\title{
Magnetooptics in Layered Nonlinear Structures
}

\author{
A.D. BoARDMAN AND M. XIE \\ Photonics and Nonlinear Science Group, Joule Physics Laboratory \\ Institute of Materials Research, University of Salford, Salford, M5 4WT, UK
}

\begin{abstract}
The magnetooptics of an asymmetric layered waveguide structure is discussed to investigate the possibility of creating new devices that combine both nonreciprocity and nonlinearity. After addressing some fundamental magnetooptic properties and the kind of materials needed, a straightforward envelope theory is given that exploits the Voigt effect instead of the more obvious Faraday effect. It is emphasised that this choice leads to desirable design features. It is proposed that the introduction of an applied magnetic field to a waveguide structure can be achieved through the use of thin current-carrying strips. It is emphasised that the strips can be arranged to any degree of complexity and that a high degree of control over spatial soliton dynamics can be achieved. Spatial soliton light beams are selected because they can be generated, within a waveguide, in a stable form. The attractive features of using the type of magnetooptic waveguide investigated here are highlighted with examples and it is concluded that the formats proposed can be used for the fully integrated isolator capacity that modern laser systems demand.
\end{abstract}

PACS numbers: 42.65.Tg, 42.65.Wi, 42.25.Fx, 78.20.Ls

\section{Introduction}

Magnetooptics involves the behaviour of light in a dielectric medium exposed to an applied magnetic field. It is a phenomenon that deeply involves the polarization state of light [1], which is a measure of its vector property. To control this is to embrace a positive use of magnetooptically-driven complexity that introduces forced gyrotropy [2] into a waveguide structure. In a magnetooptic material, a plane wave with a spatial variation $\exp (-\mathrm{i} k \cdot r)$, where $r=(x, y, z)$ and $k$ is a wave vector, is associated with a displacement vector $\boldsymbol{D}$, in the form [3]:

$$
\boldsymbol{D}=\varepsilon_{0}[\varepsilon \cdot \boldsymbol{E}+\mathrm{i} \boldsymbol{g} \times \boldsymbol{E}],
$$


where $\varepsilon$ is the dielectric tensor in the absence of a magnetic field and a gyration vector $\boldsymbol{g} \propto \boldsymbol{H}_{\mathrm{app}}$ is introduced through $\boldsymbol{H}_{\mathrm{app}}$, the applied magnetic field vector. From the structure of (1), it is apparent that $\boldsymbol{g} \times \boldsymbol{E}$ is normal to $\boldsymbol{E}$, so that the dielectric tensor associated with a magnetooptic material will have off-diagonal elements. It is also the case that $\boldsymbol{g}$ does not depend upon which way the light wave is going. This means that it continues to act even after the wave has been forced to change its direction by a reflection. Hence the physical system described by (1) is nonreciprocal.

Although gyrotropy is the word adopted in Western literature to describe a physical situation involving nonreciprocity, it is important to note that gyrotropy has also been adopted in Soviet literature [4] to describe chirality. In fact, there is nothing wrong in this because gyrotropy comes from the Greek word gyros meaning circle, so gyrotropy is a generic description of any rotation of the vibration plane of light.

If a plane, linearly polarised, light wave passes once through a block of magnetooptic material that is in a magnetooptic state, due to an applied magnetic field along the propagation direction, then the plane of polarisation, or vibration $[1,2]$, will be rotated. If this wave is reflected back through the material then, as implied by (1) and the previous discussion, the plane will continue to rotate in the same direction. Faraday discovered this so it is now called the Faraday effect. It describes the propagation of light parallel to, or antiparallel to, an applied magnetic field. Another magnetooptic effect is called the Voigt, or Cotton-Mouton effect, also eponymously named after their co-discoverers. Their discoveries go back to the beginning of the last century when Voigt observed double refraction as white light passed through sodium vapour that was exposed to a strong magnetic field, applied perpendicular to the propagation direction. Within a year of this discovery, Cotton and Mouton also found double refraction, for the same applied magnetic field-propagation direction arrangement. This time, however, the effect was a thousand times greater, because they passed white light through nitrobenzene. It is helpful to note, at this stage of the discussion, that the kind of double refraction given by the Cotton-Mouton magnetooptic effect is also like the signature of the Kerr electrooptic effect. Furthermore, since either of the names Voigt or Cotton-Mouton can be used without loss of clarity, Voigt will be the name of choice in this paper.

For a bulk medium, the signature of the Faraday effect is the rotation of the plane of polarisation of the electric field vector and this can be resolved into two counter-rotating circularly polarised waves. Each wave is characterised by a refractive index that is proportional to the applied magnetic field. A reversal of the field interchanges the indices and it is this property that makes the Faraday effect nonreciprocal. On the other hand, the bulk medium Voigt effect is reciprocal because the birefringence is now proportional to the square of the applied magnetic field. This property seems to be a major setback for the use of propagation 
transverse to an applied magnetic field in the design of integrated magnetooptic structures. In fact, this is not the case, since the Voigt effect is nonreciprocal in an asymmetric waveguide and this will be discussed later on.

Recent advances in material science now make many integrated formats possible, so the real issue of nonreciprocity is how to fabricate applications that give full realisation to their potential. They have to depend heavily upon waveguide design, or some other ideas, like post-fabrication processing, to overcome weaknesses created by fabrication tolerances [5]. The global trends, however, show that integrated [6] magnetooptic devices are poised to become pivotal to many applications.

The integration of magnetooptic devices with semiconducting substrates that include [7-9] active devices, such as lasers, detectors, amplifiers and nonlinearity is very desirable and the nonlinear direction has been clearly mapped out by applying an external magnetic field to a waveguide containing third-order optically nonlinear material and linear magnetooptic elements [10-15].

Magnetooptic materials are easily created $[6,16]$ but to overcome domain effects, they require the application of an external magnetic field [17] and it is films of material, like yttrium iron garnet (YIG) that have generally attracted attention $[5,6]$. As can be seen in (1), $\boldsymbol{g} \times \boldsymbol{E}$ is perpendicular to $\boldsymbol{E}$ so an external magnetic field creates off-diagonal elements in the permittivity tensor that are proportional to the induced magnetisation. These elements are defined here as $\pm \operatorname{in} n_{\mathrm{m}}^{2} Q$, where $n_{\mathrm{m}}$ is the normal linear refractive index of the magnetic material that appears in the diagonal elements of the dielectric tensor and $Q$ is a magnetooptic parameter, proportional to the applied magnetic field. In general, $Q$ is a spatial distribution and is a relatively complicated function of the magnetisation of the material. Another consideration is that $Q$ often vanishes, in the absence of any applied magnetic field, leaving no permanent magnetisation, because of magnetic domain effects i.e. upon switching off the applied magnetic field, the net magnetisation can return to zero. This may not always be the case, in practice, but it does happen quite naturally [17]. Added to this may be a concern that the desired length of device may be compromised by attenuation. This is actually not such a problem because integrated-optical circuit, magnetooptic, devices will use rather weakly absorbing materials, such as layers of $(\mathrm{LuNiBi})_{3}(\mathrm{FeAl})_{5} \mathrm{O}_{12}$ deposited upon $\mathrm{Gd}_{3} \mathrm{Ga}_{5} \mathrm{O}_{12}$ substrates. Also, the substitution by cerium of the rare-earth garnet [6] yields large magnetooptic coefficients. It is also accepted that the widespread use of bismuth and aluminium doping, reduces attenuation to even more acceptable levels. All of these comments serve to highlight evidence that magnetooptic devices are very promising candidates for switching and routing [5, 6, 18, 19].

Clearly, the point of using magnetooptics is to introduce extra degrees of freedom into guided-wave processes, over and above those that appear through crystal symmetry. Indeed, exploiting magnetooptic properties will produce very impressive integrated units [7-9], when compared to those based purely upon 
GaAs, or $\mathrm{LiNbO}_{3}$, technologies. To achieve this goal, the key issues promoting success must be the optimised selection of magnetooptic materials and the design of robust magnetooptic waveguides. After this is achieved, exercising the option of adding a nonlinear effect yields even greater design flexibility. As stated earlier, low loss propagation can now be expected and the classic material to use is an expitaxially grown YIG film. Looking ahead to the applications, it is desirable, therefore, to explore the feasibility of using YIG-semiconductor structures, so that amplification can be added in during propagation. Experimentally, it is always a bonus that YIG is optically transparent over the $1-5 \mu \mathrm{m}$ range of wavelengths and also that its magnetisation is saturable by small, easily generated, magnetic fields. The downside is that YIG cannot be deposited [16] epitaxially onto a material like GaAs or its derivatives, which is exactly the kind of material that could be selected to provide amplification, through carrier drift mechanisms. Even so, buffer layers or diluted magnetic semiconductors [16] can be used instead, without loss of performance, and it has been pointed out recently that YIG can actually be "attached" to GaAs [20].

The really important applications for magnetooptic devices include pivotal activity in laser control [7] through isolators and a lot of effort has gone into production and design [5], compatible with low-loss nonreciprocal circuits. Both isolators and circulators are required as part of optical communication systems, with circulators, in particular, facilitating routing capabilities and two-way communications. In this context, a lot of TE-TM conversion work has appeared in the linear domain but phase-matching remains a difficult problem to overcome. Also manufacturing tolerances [5] can reduce device performance to unacceptable levels. Another comment about TE-TM conversion is that it relies too much upon the deployment of the traditional Faraday effect, so another way forward has become popular $[5,18,19]$. This uses nonreciprocal modes in the kind of asymmetric waveguide discussed earlier, in which the magnetic field is perpendicular to the propagation direction and lies in the waveguide plane. A simple analysis of its dispersion properties $[13,14]$ reveals that the guided linear TE modes are not affected, to first-order, by an applied magnetic field, but, on the other hand, TM guided modes have amplitudes with a gradient along the propagation direction proportional to the applied magnetic field. This conclusion has led to a preference for the Voigt effect, over the Faraday effect because there is no need now to address phase-matching considerations, since any coherence exponential factors involving the birefringence are now entirely absent. The asymmetric guide, therefore, is the designers' choice for applications like isolators.

All conventional work on isolators and circulators has, up to now, ignored the question of optical nonlinearity. This is rather surprising, at first sight, since the magnetooptic community is currently intensely interested in nonlinear magnetooptic. The explanation lies in the focus of the magnetism community upon the measurement of the Kerr rotation of the plane of vibration of the light wave, 
reflected from ferromagnetic surfaces. There the attention is directed to the second-harmonic generation because it was discovered, recently [21] that a giant nonlinear Kerr rotation can be observed in the second-harmonic wave, created by reflection off low-dimensional structures (thin films), using a beam from a Ti:sapphire laser. This second-harmonic excitation is generated by symmetry-breaking at the surface so this is not the unusual part. The exciting effect is that the nonlinear Kerr rotation is an order of magnitude larger than anything that can be achieved in the linear domain. This enhancement of the Kerr rotation is aided by magnetically-induced elements of the nonlinear susceptibility tensor. The activity surrounding the Kerr rotation effects is in sharp contrast, therefore, to the guided wave work reported here.

\section{Basic theory}

The paradigm asymmetrical planar waveguide used here is shown in Fig. 1. It supports a spatial soliton beam of light, propagating along a $z$-axis that is perpendicular to a magnetisation created by a magnetic field applied along the $x$-axis. The waveguide has an upper air boundary and consists of a nonlinear layer of thickness $d$, attached to a thick magnetised substrate. The solitons are one-dimensional but are stable because the waveguide is sufficiently thin to prevent the beam diffracting along the $y$-direction.

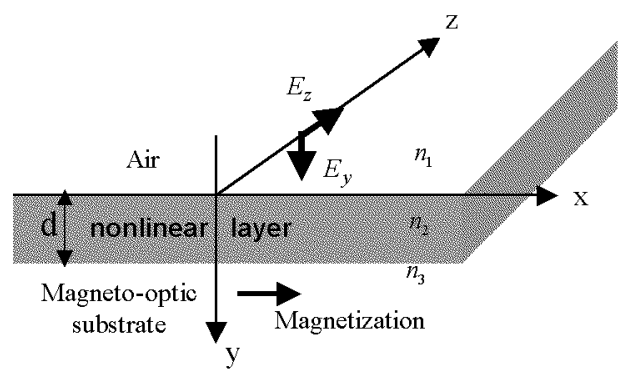

Fig. 1. Sketch of a planar waveguide structure, unbounded in the $\pm x$ directions.

The TM wave has an electric field vector

$$
\boldsymbol{E}=A(x, z)\left[\xi_{y}(y) \hat{y}+\xi_{z}(y) \hat{z}\right] \exp \left(-i \frac{\omega}{c} \beta z\right) \exp (i \omega t),
$$

in which $\xi(y)$ is the vector modal field shape, $\beta$ is the effective refractive associated with the TM wave, $\omega$ is an angular frequency, $c$ is the vacuum velocity of light and $A(x, z)$ is a slowly varying amplitude envelope.

$A(x, z)$ satisfies the following envelope equation [10-15]:

$2 \mathrm{i} \frac{c}{\omega} \frac{\partial A}{\partial z}=\frac{c^{2}}{\omega^{2} \beta} \frac{\partial^{2} A}{\partial x^{2}}+2 \bar{\varepsilon}_{y z} A+\bar{\chi}_{\mathrm{m}} A|A|^{2}$. 
To arrive at (3), the magnetooptic and nonlinear properties are taken as perturbations and then an average over the whole waveguide is taken. This results in $\bar{\chi}_{\mathrm{m}}$ being an average of the self-phase modulation coefficient and $\bar{\varepsilon}_{y z}$ being an average of the $\varepsilon_{y z}$ component of the linear magnetooptic dielectric tensor i.e.

$$
\begin{aligned}
& \bar{\varepsilon}_{y z}=\frac{c}{\omega \beta^{2}} \frac{\int \varepsilon_{y z} \xi_{y} \frac{\partial \xi_{y}}{\partial y} \mathrm{~d} y}{\int\left(\left|\xi_{y}\right|^{2}+\left|\xi_{z}\right|^{2}\right) \mathrm{d} y}, \\
& \bar{\chi}_{\mathrm{m}}=\frac{3}{4} \frac{\int 2 \chi_{x x y y}\left(\left.\varepsilon_{y}\right|^{2}+\left|\varepsilon_{z}\right|^{2}\right)^{2} \mathrm{~d} y+\int \chi_{x y y x}\left(\xi_{y}^{2}+\xi_{z}^{2}\right)\left(\xi_{y}^{* 2}+\xi_{z}^{* 2}\right) \mathrm{d} y}{\beta_{2} \int\left(\left|\xi_{y}\right|^{2}+\left|\xi_{z}\right|^{2}\right) \mathrm{d} y},
\end{aligned}
$$

in which $\chi_{x x y y}, \chi_{x y y x}$ are third-order nonlinear susceptibility tensor elements.

This equation has spatial solitary wave solutions (loosely called solitons) but a constant $\bar{\varepsilon}_{y z}$ does nothing except to cause the soliton to experience an additional constant phase shift. This is easy to see because a simple transformation will permit the $2 \bar{\varepsilon}_{y z} A$ and the $2 \mathbf{i} \frac{c}{\omega} \frac{\partial A}{\partial z}$ terms to be combined. Secondly, $\bar{\varepsilon}_{y z}$ contains the gradient $\partial \xi_{y} / \partial y \propto \xi_{z}$ in the numerator. Hence, a significant refractive index step is permitted at the boundary, in order to achieve a reasonable value of longitudinal electric field component $E_{z}$.

Equation (3) can be transformed to a dimensionless state through the definitions

$$
A=\frac{c}{\omega D_{0}} \frac{\sqrt{2}}{\sqrt{\beta_{2} \overline{\chi_{\mathrm{m}}}}} \psi, \quad \nu=2 \beta_{2} \frac{\omega^{2}}{c^{2}} D_{0}^{2} \bar{\varepsilon}_{y z}, \quad x \rightarrow D_{0} x^{\prime}, \quad z \rightarrow L_{D} z^{\prime} .
$$

Here $L_{D}=2 \beta_{2} \frac{\omega}{c} D_{0}^{2}$ is called the diffraction, or Rayleigh, length and is the distance over which diffraction alone causes a beam to double in size. After the dashes are dropped, for convenience, the basic envelope equation becomes

$$
-\mathrm{i} \frac{\partial \psi}{\partial z}+\frac{\partial^{2} \psi}{\partial x^{2}}+\nu \psi+2|\psi|^{2} \psi=0
$$

Typically, $\bar{\chi}_{\mathrm{m}}=5.5 \times 10^{-11} \mathrm{~m}^{2} \mathrm{~V}^{-2}, D_{0} \approx 10 \mu \mathrm{m}, \beta=2.67, d=0.3 \mu \mathrm{m}$, $n_{1}=1, n_{2}=3.32, n_{3}=2.3, L_{D}=2.16 \mathrm{~mm}$, and $\nu=1.6$. This data corresponds to an $\mathrm{AlGaAs}$ film attached to a simple magnetic garnet substrate, like YIG, or a more complex magnetic garnet like $\mathrm{Y}_{2.5} \mathrm{Ce}_{0.5} \mathrm{Fe}_{5} \mathrm{O}_{12}$.

The Lagrangian density that will generate Eq. (7), after the application of the Euler-Lagrange equations, is

$$
L=\frac{\mathrm{i}}{2}\left(\psi^{*} \frac{\partial \psi}{\partial z}-\frac{\partial \psi^{*}}{\partial z} \psi\right)-\left|\frac{\partial \psi}{\partial z}\right|^{2}+|\psi|^{4}+\nu|\psi|^{2}
$$

and a suitable trial function to use in the formulation of a variational calculation is

$$
\psi=\eta(z) \operatorname{sech}\left\{\eta(z)\left[x-x_{0}(z)\right]\right\} \exp \left\{i \frac{\xi(z)}{2}\left[x-x_{0}(z)\right]+\mathrm{i} \phi(z)\right\} .
$$

In this function, $x_{0}(z)$ is now a $z$-dependent location of the centre of the localised state (soliton), $\eta(z)$ is the amplitude, $\xi(z)$ is the inclination of the spatial soliton 
beam axis to the propagation direction and $\phi(z)$ is a phase factor. The average Lagrangian [22] is

$$
\begin{aligned}
L= & \int_{-\infty}^{\infty} L \mathrm{~d} x=\eta\left(\xi \frac{\partial x_{0}}{\partial z}-2 \frac{\partial \phi}{\partial z}\right)+\frac{2}{3} \eta^{3}-\frac{\xi^{2}}{2} \eta \\
& +\eta^{2} \int \nu(x) \operatorname{sech}^{2}\left(x-x_{0}\right) \mathrm{d} x
\end{aligned}
$$

where the possibility that the magnetooptic parameter $\nu$ could be a function of $x$, the transverse coordinate, is admitted for the first time.

The application of the Euler-Lagrange equations, and the deployment of a first integral over $x$, leads to the energy equation

$$
\frac{1}{2}\left(\frac{\mathrm{d} x_{0}}{\mathrm{~d} z}\right)^{2}-\int \nu(x) \operatorname{sech}^{2}\left(x-x_{0}\right) \mathrm{d} x=\text { const. }
$$

Hence, a potential function $U\left(x_{0}\right)$ controls the soliton dynamics, where

$$
U\left(x_{0}\right)=-\int \nu(x) \operatorname{sech}^{2}\left(x-x_{0}\right) \mathrm{d} x .
$$

Experimentally, the issue is whether a credible function $\nu(x)$ can be economically created, so that a useful potential barrier/well $U\left(x_{0}\right)$ can be brought into existence.

\section{Soliton control with electrode patterns}

What is needed is the placement of an electrode structure on the upper surface of the guide (the nonlinear/air interface), to create a magnetic field that drops rapidly towards zero in the $( \pm x)$-directions. In this way, a magnetic field will be created that induces a magnetisation that is saturated, or otherwise, over a limited region of the magnetic substrate. Typically, the magnetooptic, insulating, material will experience a saturation of its magnetisation at about 300 Oe. Since $\nu(x)$ is proportional to the magnetisation, it means that $\nu(x)$ saturates to a maximum value. For many materials, a simple hyperbolic tangent function models the observed variation of magnetisation very well. It will be assumed, therefore, that $\nu=\tanh \left(B H / H_{\mathrm{s}}\right)$ where $A$ and $B$ are just empirical constants, selected to make tanh $\rightarrow 1$, in the region of high magnetic field $H$, which has the saturation value $H_{\mathrm{s}}$.

Electrode structures have been used $[18,19]$ in the past to create linear couplers, which consist of silver electrodes $20 \mu \mathrm{m}$ wide, carrying as little as $1 \mathrm{~mA}$ of electric current. The magnetic field that they produce can be accurately approximated by that produced by an infinitely long thin wire, or a set of wires. Some experimentally accessible electrode structures are shown in Fig. 2 and the coordinate system for a single wire system is shown in Fig. 3. In the latter case, the modulus of the magnetic field at the boundary is 


$$
H=\frac{I}{2 \pi \sqrt{x^{2}+d^{2}}} .
$$

For $I=60 \mathrm{~mA}$ and $d=0.3 \mu \mathrm{m}$ the maximum field directly under the wire is $H(0)=I / 2 \pi d=400 \mathrm{Oe}$. If the magnetic substrate becomes saturated at $300 \mathrm{Oe}=$ $23.87 \times 10^{3} \mathrm{~A} \mathrm{~m}^{-1}$, then the $x$-position at which the magnetic field is enough to saturate the magnetic material is $x=0.27 \mu \mathrm{m}$. For $\nu=A \tanh \left(B H / H_{\mathrm{s}}\right)$, with $A=1.6$, for example, Fig. 4 shows a plot of the quantity $\nu \sin \gamma$ that participates in saturating the magnetisation.
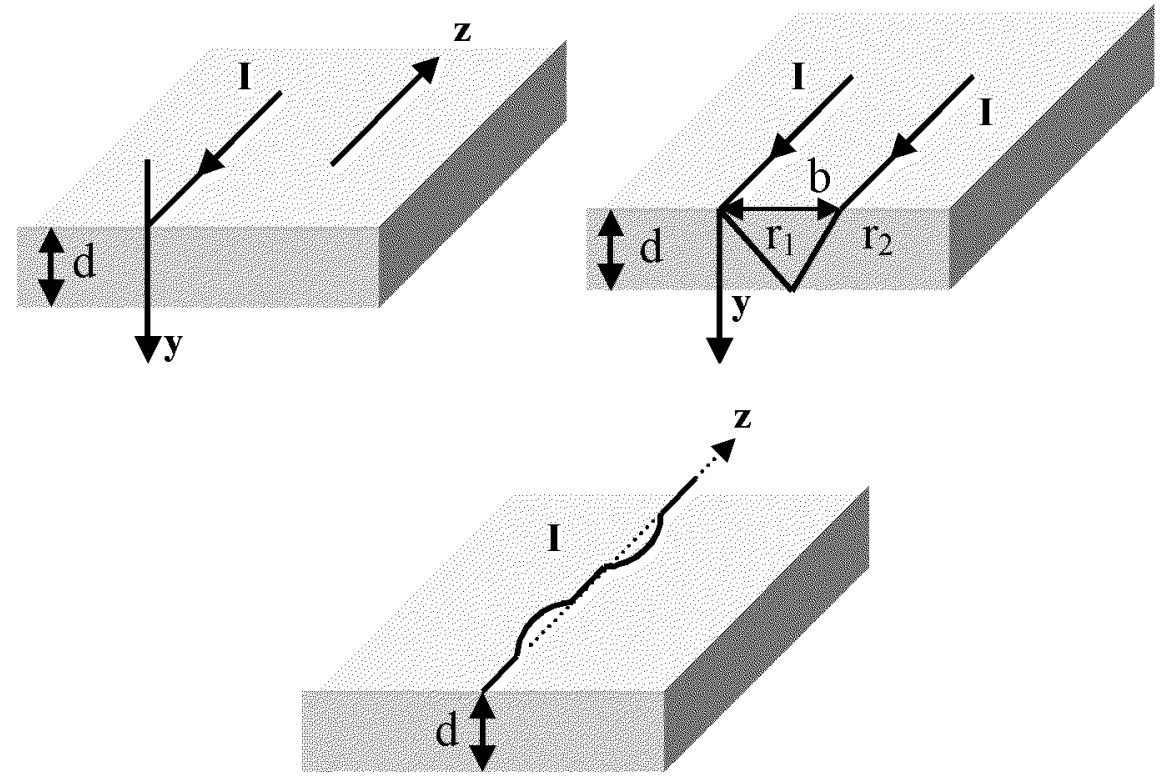

Fig. 2. Some current strip-line configurations. The current $I$ flows along the top surface in thin metallic strips.

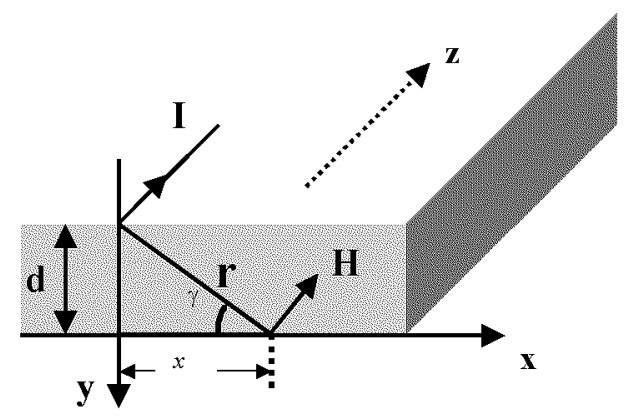

Fig. 3. Coordinate system for a single wire. 


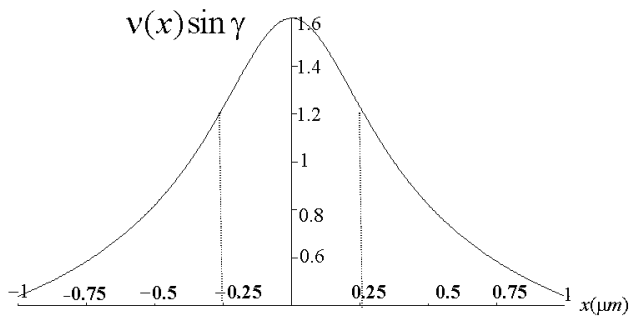

Fig. 4. Magnetooptic parameter distribution for a single current strip wire.

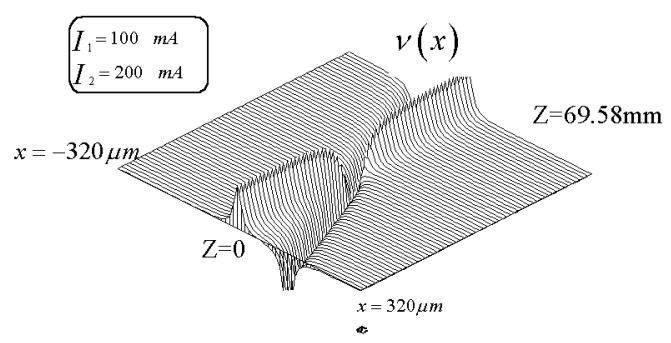

Soliton dynamics
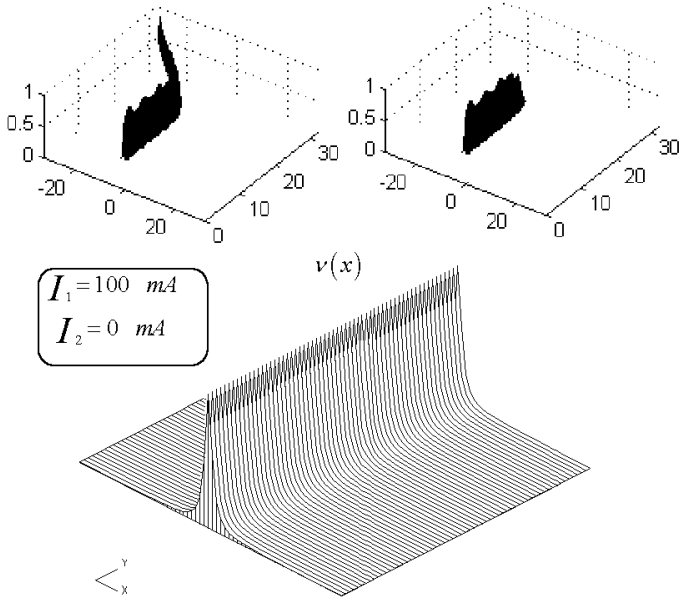

Fig. 5. Double current strip electrode.

For a two parallel wire electrode structure, in which the currents are flowing in opposite directions, the soliton dynamics are interesting. The $\nu(x)$ shown in Fig. 5 , for $I_{1}=100 \mathrm{~mA}, I_{2}=200 \mathrm{~mA}$, has a complicated shape and the $(x, z)$ scales reveal a transverse $\mu \mathrm{m}$ domain that is attractive for integration. The propagation is in the mm domain, so the soliton dynamics are clearly accessible experimentally. Figure 5 shows that a soliton encountering the structure shown bounces off it. Not 
shown in Fig. 5 is the fact that for other current values, such as $I_{1}=100 \mathrm{~mA}$, $I_{2}=140 \mathrm{~mA}$, there can be some energy splitting. If one current is switched off it can be seen that $\nu(x)$ becomes just a simple regular function. In principle, there is no limit to the well/barrier structures that can be created, and the fabrication processes are well within the capabilities of current technology. Even curved strips can be used.

\section{Conclusions}

The basic idea of including magnetooptic layers, claddings or substrates into the standard planar waveguide format has been investigated. It is attractive for integrated optics because the aim is to produce an integrated optics "chip-level" format that will participate in, and control, all-optical processing operations in the future. The use of electrode patterns on nonlinear magnetooptic materials is an attractive way forward, if spatial soliton beams, trapped in a waveguide, are used. They exploit diffraction, which is always rather strong and operates over only the order of $\mathrm{mm}$ in the propagation direction and the order of $\mu \mathrm{m}$ in the transverse direction. The deployment of spatial solitons, therefore, fits the "chip" format very well. In addition, it is perfectly feasible to scale everything down to the nanotechnology level. Controlling soliton dynamics through magnetooptics is a powerful idea because the electrode structures are strips of metal that can easily be manipulated and produced in any desired format. Even the simplest of them gives such an impressive degree of control that superimposed, or buried, electrode structures will become a feature of all-optical chip technology, in the near future. The accessibility of outstanding magnetooptic materials, which can be sculptured to within acceptable tolerance limits [5] is a major driver in linear magnetooptics. This fact should encourage experimental work on nonlinear guided magnetooptics.

\section{References}

[1] E. Hecht, A. Zajac, Optics, Addison-Wesley, Reading 1974.

[2] J. Petykiewicz, Wave Optics, Kluwer, Dordrecht 1992.

[3] B.E. Saleh, M.C. Teich, Fundamentals of Photonics, Wiley, New York 1991.

[4] I.V. Lindell, A.H. Sihvola, S.A. Tretyakov, A.J.J. Viitanen, Electromagnetic Waves in Chiral and Bi-Isotropic Media, Artech House, Boston 1994.

[5] N. Bahlmann, M. Lohmeyer, O. Zhuromskyy, H. Dotsch, P. Hertel, Opt. Commun. 161, 330 (1999).

[6] A.K. Zvezdin, V.A. Kotov, Modern Magneto-optics and Modern Magnetooptic Materials, Institute of Physics, Bristol 1997.

[7] W. Zaets, K. Ando, IEEE Phot. Tech. Lett. 11, 185 (2001).

[8] J. Hammer, J.H. Abeles, D.J. Channin, IEEE Phot. Tech. Lett. 9, 631 (1997). 
[9] O. Zhuromskyy, M. Lohmeyer, N. Bahlmann, H. Dotsch, P. Hertel, A.F. Popkov, J. Lightwave Tech. 17, 1200 (1999).

[10] A.D. Boardman, K. Xie, Phys. Rev. Lett. 76, 4591 (1995).

[11] A.D. Boardman, K. Xie, Phys. Rev. E 55, 1899 (1997).

[12] A.D. Boardman, K. Xie, J. Opt. Soc. Am. B 15, 3102 (1997).

[13] Soliton-driven Photonics, Eds. A.D. Boardman, A.P. Sukhorukov, Kluwer, Boston 2001.

[14] A.D. Boardman, M. Xie, J. Opt. B, Quantum Semiclass. Opt. 3, S244 (2001).

[15] A.D. Boardman, M. Xie, J. Opt. Soc. Am. B 19, 719 (2002).

[16] Magnetooptics, Eds. S. Sugano, N. Kojima, Springer, Berlin 2000.

[17] M.S. Sodha, N.C. Srivastava, Microwave Propagation in Ferrimagnetics, Plenum, New York 1981.

[18] T. Mizumoto, H. Chihara, N. Tokui, Y. Naito, Electron. Lett. 26, 199 (1990).

[19] T. Mizumoto, Y. Naito, IEEE Trans. MTT 30, 922 (1982).

[20] M. Levy, R.M. Osgood, A. Kumar, Appl. Phys. Lett. 71, 2617 (1997).

[21] W. Hübner, Physics World 8, 21 (1995).

[22] G.B. Whitham, Linear and Nonlinear Waves, Wiley, New York 1974. 